\title{
Typhlitis in Acute Childhood Leukemia
}

\author{
Esma Altınel Nese Yarali Pamir Isık Ali Bay Abdurrahman Kara \\ Bahattin Tunc \\ Department of Pediatric Hematology, Dr. Sami Ulus Children's Hospital, Ankara, Turkey
}

\section{Key Words}

Typhlitis $\cdot$ Leukemia $\cdot$ Children

\begin{abstract}
Objective: To review our experience with typhlitis among children treated for acute leukemia. Material and Methods: The medical records of children with acute leukemia and typhlitis between 2006 and 2009 were reviewed for demographics and symptoms, and for microbiological and imaging findings. Results: In the 75 children with acute leukemia - 54 with acute lymphoblastic leukemia (ALL) and 21 with acute myeloid leukemia (AML) - there were 10 episodes of typhlitis (4.5\%) that developed during 221 periods of severe neutropenia. The cumulative risk of typhlitis was $7.4 \%$ in patients with ALL and $28.5 \%$ in patients with AML. Frequent symptoms were: abdominal pain and tenderness (100\% each); fever and nausea (90\% each); emesis (80\%); diarrhea (50\%), and hypotension, peritonitis and abdominal distension (10\% each). The median duration of symptoms was 6 days (range: 2-11 days), and that of neutropenia 14 days (range: 3-25 days). All patients were treated medically and none surgically. Two patients died because of typhlitis and sepsis. Conclusions: In our study, the rate of typhlitis among leukemic children was 4.5\%; however, the mortality rate was $20 \%$. Thus, rapid identification and timely, aggressive medical intervention are necessary to reduce the morbidity and mortality from typhlitis.

Copyright $\odot 2011$ S. Karger AG, Basel
\end{abstract}

\section{Introduction}

Typhlitis or neutropenic enterocolitis is a severe complication induced by chemotherapy usually involving the cecum, colon and a terminal part of the ileum that may progress to necrosis, hemorrhage, perforation and septicemia $[1,2]$. The initial symptoms are not specific and usually occur during the period of neutropenia, with rapid improvement after neutrophil recovery. The clinical features include fever, crampy abdominal pain, tenderness in the right lower quadrant, diarrhea, nausea and emesis $[3,4]$. The exact pathogenesis of typhlitis is unknown. Gut mucosal ulcerations may result from direct drug-related cytotoxicity and/or neutropenia itself following microbial invasion of the bowel wall $[1,5]$. Diagnosis is most often based on radiological findings. The disease responds to conservative management. Surgical intervention is generally reserved for specific complications or where another surgical pathologic condition cannot reasonably be ruled out $[2,6]$.

There is a paucity of published data on the incidence, outcome and risk factors of typhlitis in children with leukemia. In this retrospective study, we investigated clinical, microbiologic and radiologic findings, and the outcome of typhlitis in children with leukemia.

\section{KARGER \\ Fax +41613061234 \\ E-Mail karger@karger.ch}

www.karger.com
(C) 2011 S. Karger AG, Basel

1011-7571/12/0211-0036\$38.00/0

Accessible online at:

www.karger.com/mpp
Dr. Nese Yarali

Dostkent sitesi No. 25

Çayyolu-Çankaya

TR-06810 Ankara (Turkey)

Tel. +90 312596 9994, E-Mail neseyarali@yahoo.com 
Table 1. Frequency of clinical symptoms

\begin{tabular}{lc}
\hline Symptoms & Frequency \\
\hline Abdominal pain & $10(100 \%)$ \\
Fever & $9(90 \%)$ \\
Abdominal tenderness & $10(100 \%)$ \\
Peritonism & $1(10 \%)$ \\
Abdominal distension & $1(10 \%)$ \\
Vomiting & $8(80 \%)$ \\
Nausea & $9(90 \%)$ \\
Diarrhea & $5(50 \%)$ \\
Hypotension & $1(10 \%)$ \\
\hline
\end{tabular}

\section{Material and Methods}

Medical records of 75 children with acute leukemia treated between 2006 and 2009 at the Department of Pediatric Hematology in Dr. Sami Ulus Children's Hospital, Ankara, Turkey, were reviewed to identify patients who were diagnosed with febrile neutropenia and typhlitis. Altogether there were 221 episodes of febrile neutropenia among 54 patients with acute lymphoblastic leukemia (ALL) and 21 patients with acute myeloid leukemia (AML). Neutropenia was defined as a neutrophil count lower than $0.5 \times 10^{9} / 1$, and typhlitis was defined as the presence of the clinical triad of abdominal pain, fever and neutropenia or imaging signs (thickened bowel wall) plus two of the clinical features. Case notes were analyzed for age, sex, classification of leukemia, chemotherapy regimens, clinical findings, imaging results, duration and management of typhlitis.

Patients were treated with either a combination of cytosine arabinoside, etoposide and idarubicin (BFM 93 induction), or a combination of prednisone, vincristine, L-asparaginase, daunomycin, etoposide and cytosine arabinoside (St. Jude Total XIII induction protocol). All patients had blood cultures, ultrasound (US) images and plain abdominal X-rays. All statistical analyses were performed with the SPSS 11.5 software system for Windows.

\section{Results}

Typhlitis developed after intensive chemotherapy in 5 patients with AML, 4 patients with ALL and 1 patient with biphenotypic leukemia. The cumulative incidence of typhlitis was $7.4 \%$ in patients with ALL and $28.5 \%$ in patients with AML or biphenotypic leukemia. The pertinent features of the 10 patients with typhlitis are summarized in table 1 . The median age was 7.5 years (range: $3-13$ years). All patients had abdominal pain and tenderness. The median duration of symptoms was 6 days (range: 2-11 days), and that of neutropenia was 14 days (range: 3-25 days).

Typhlitis in Acute Childhood Leukemia
Typhlitis developed in 4 patients with AML shortly after BFM 93 induction, in 3 with ALL during the St. Jude Total XIII induction protocol, in 1 promyelocytic leukemia patient during AIDA (all-trans retinoic acid and idarubicin) induction treatment, in 1 AML patient after high-dose cytosine arabinoside, and in 1 ALL patient after high-dose methotrexate. The blood cultures of 3 patients (30\%) were positive; the organisms isolated were Escherichia coli (extended-spectrum $\beta$-lactamase positive), Candida spp. and viridans-group streptococci (table 2).

In 2 cases where US demonstrated nonspecific bowel wall thickening and could not differentiate appendicitis, CT was performed, and marked hypodense ileocecal wall thickening and increased mucosal enhancement was demonstrated. The abdominal X-ray showed small bowel dilatation in 1 case, and in 3 cases thickened air-filled loops of bowel. In others, the abdominal X-ray was normal. Six patients had a transmural inflammatory pattern of the thickened bowel wall, with the entire ileocecal region involved. In 4 cases with normal US, typhlitis was diagnosed based on clinical features and/or findings by abdominal X-ray.

The 10 patients were treated with multiple antibiotics, intensive fluid replacement and bowel rest. None of them was treated surgically. The antibiotic treatment usually started with a $\beta$-lactam combined with aminoglycoside. Metronidazole was added for 8 patients, and vancomycin for 5 patients. For the patient with Candida sepsis, liposomal amphotericin B was added as antifungal treatment. Granulocyte colony-stimulating factor was given to 3 patients to shorten the time of neutropenia. Two patients who had thick bowel walls $(0.5 \mathrm{~cm}$ and $1.2 \mathrm{~mm}$, respectively) assessed by US died of sepsis and multiorgan failure.

\section{Discussion}

The incidence of typhlitis of $10 \%$ in our study was higher than the reported range of $2.6-6.7 \%$ in patients hospitalized for hematological malignancies $[2,6,7]$. The cumulative incidence of typhlitis was $7.4 \%$ in patients with ALL and 28.5\% in those with AML. Various factors play a role in the pathogenesis of typhlitis. Apart from direct damage to the mucosa by leukemic infiltrates, toxic effects of chemotherapeutic agents are responsible for the pathogenesis $[3,8,9]$. Several drugs are known to be associated with oral mucositis or gastrointestinal toxicity including diarrhea [4]. Other factors include a combination of local bacterial and fungal infections, necrosis of 
Table 2. Characteristic findings in patients with typhlitis

\begin{tabular}{|c|c|c|c|c|c|c|c|c|c|}
\hline $\begin{array}{l}\text { Case } \\
\text { No. }\end{array}$ & $\begin{array}{l}\text { Age, } \\
\text { years }\end{array}$ & Sex & Diagnosis & Treatment & $\begin{array}{l}\text { WBC, } \\
\times 10^{9} / 1\end{array}$ & $\begin{array}{l}\text { ANC, } \\
\mathrm{n} / \mathrm{mm}^{3}\end{array}$ & Blood culture & Imaging & Outcome \\
\hline 1 & 11 & $\mathrm{~F}$ & AML & BFM 93 (induction) & 0.6 & $<500$ & - & X-ray, US ${ }^{1}$ & resolution \\
\hline 3 & 10 & $\mathrm{M}$ & ALL & St. Jude T XIII (induction) & 1.4 & $<500$ & - & X-ray, US & resolution \\
\hline 4 & 6 & $\mathrm{~F}$ & AML & BFM 93 (induction) & 0.7 & $<500$ & Candida spp. & X-ray ${ }^{3}$, US $^{1}$ & resolution \\
\hline 5 & 4 & $\mathrm{M}$ & ALL & St. Jude T XIII (induction) & 0.3 & $<500$ & E. coli (ESBL) & X-ray, US & resolution \\
\hline 7 & 7 & $\mathrm{M}$ & ALL & St. Jude T XIII (induction) & 67.7 & $<500$ & - & X-ray ${ }^{2}, \mathrm{US}^{1}$ & death \\
\hline 8 & 13 & $\mathrm{M}$ & AML & AIDA (induction) & 1.9 & $<500$ & - & X-ray ${ }^{2}$, US & resolution \\
\hline 9 & 10 & $\mathrm{M}$ & AML & BFM 93 (induction) & 0.8 & $<500$ & - & $\mathrm{X}$-ray, $\mathrm{US}^{1}, \mathrm{CT}$ & resolution \\
\hline 10 & 5 & $\mathrm{M}$ & ALL & St. Jude T XIII (HDMTX) & 0.4 & $<500$ & - & X-ray, US ${ }^{1}$ & resolution \\
\hline
\end{tabular}

$\mathrm{WBC}=$ White blood cells; $\mathrm{ANC}=$ absolute neutrophil count; $\mathrm{T}=$ total; HAM = high-dose cytosine arabinoside plus mitoxantrone; AIDA = all-trans retinoic acid plus idarubicin; HDMTX = high-dose methotrexate; ESBL = extended-spectrum $\beta$-lactamase .

${ }^{1}$ Thickened bowel wall more than $5 \mathrm{~mm}$ with entire ileocecal region involved. ${ }^{2}$ Air-filled loops of bowel. ${ }^{3}$ Ileus.

the mural wall, mucosal hemorrhage, stasis of bowel contents with epithelial erosion and mucosal ischemia from sepsis-induced hypotension $[2,6,10]$. In recent years, with the use of more intensive chemotherapy regimens in hematologic malignancies especially after autologous and allogeneic stem cell transplantations, a higher incidence of neutropenic colitis has been noted [2,11]. Also certain types of solid tumor such neuroblastoma, Ewing sarcoma, osteosarcoma, rhabdomyosarcoma and Wilms' tumor have been reported to predispose patients to development of typhlitis $[4,6]$. Intensive chemotherapy, such as high-dose methotrexate or cytarabine and induction treatment, preceded the onset of symptoms in all our patients.

The typical clinical manifestations are the triad of abdominal pain or tenderness, fever and neutropenia. All usually improve after neutrophil recovery [12]. In our study, abdominal pain and tenderness was found in all patients (100\%), followed by fever and nausea in $90 \%$, emesis in $80 \%$, diarrhea in $50 \%$, and hypotension, peritonism and abdominal distension in $10 \%$, which is basically within the range reported by McCarville et al. [4]. The median duration of symptoms of 6 days and the median duration of neutropenia of 14 days were also similar to those reported by McCarville et al. [4]. The rate of E. coli, Candida spp. and viridans-group streptococci identified in the blood of 3 of our 10 patients was similar to that of bacteremia (E. coli, Klebsiella spp., Pseudomonas spp. and viridans-group of streptococci) reported previously [2].
According to the literature report, neutropenic colitis is mainly localized in the ileocecal region, although other parts of the bowel can be affected as well. A high concentration of lymphatic tissue in this area and the special anatomy of the terminal branches of the superior mesenteric artery as well as less vascular perfusion may contribute to ischemia. The cecum represents an area of relative stasis of bowel content and is easily distensible, causing high intramural pressure and insufficient blood supply [3]. Similarly, most of our patients showed a transmural inflammatory pattern of the thickened bowel wall with the entire ileocecal region involved.

The ideal imaging modality for typhlitis is controversially discussed. Abdominal X-ray may show small bowel obstruction, a paucity of gas in the right lower quadrant or a dilated, fluid-filled cecum. It can also show free intraperitoneal air, a complication of bowel perforation. However, these findings are not specific and rarely helpful in diagnosis. US and CT are more helpful [13]. CT is limited by the risk of high ionizing radiation and the need for sedation in small children, and in our study, CT was performed in only 2 cases for differentiation of appendicitis. The characteristic US features of neutropenic colitis are echogenic, asymmetric thickening of the mucosal wall with transmural inflammatory reaction and areas of different echogenicity caused by edema, necrosis and/or hemorrhages $[3,11,14]$. A mural thickness of less than $2 \mathrm{~mm}$ was considered normal, more than $2 \mathrm{~mm}$ and less than $5 \mathrm{~mm}$ was considered nonspecific bowel wall thickening, and a thickness of more than $5 \mathrm{~mm}$ was considered 
abnormal and confirming the diagnosis of neutropenic enterocolitis [3]. Abdominal X-ray and US were performed on all our patients. Six children $(60 \%)$ had ultrasonographically confirmed typhlitis; however, $40 \%$ were diagnosed with clinical and nonspecific radiologic features. Typhlitis typically affects the cecum and the ascending colon; it has been reported in the transverse colon, descending colon and even the rectum $[15,16]$. Involvement of the ileocecal region was observed in $60 \%$ of our patients.

The differential diagnoses of right lower quadrant pain include typhlitis, appendicitis, ileus and intussusception. Typhlitis usually improves with medical treatment, but other diseases need immediate surgical consult. Other acute or chronic inflammatory diseases of the ileocecal area - e.g. bacterial ileocecitis, cytomegalovirus infection, Crohn's disease, and pseudomembranous and ischemic colitis - should also be taken into account. In patients after allogeneic stem cell transplantation, one has to think of graft-versus-host disease, although this usually occurs after engraftment [2].

Optimal management of typhlitis ranges from intensive supportive care to emergency surgery. Supportive care consists of bowel rest with nasogastric decompres- sion, adequate fluid replacement and broad-spectrum antibiotics. Surgical intervention should be considered in case of persistent gastrointestinal bleeding, intestinal perforation and clinical deterioration during medical therapy [13]. Reported mortality rates range from 2.2 to $48 \%[4,13]$. The mortality rate in our study was $20 \%$. The prognosis depends on the underlying disease and on the clinical conditions of the patient. The main prognostic factors are neutrophil recovery and overall time of neutropenia. The induction phase of leukemia treatment is a very important period as in that period, the bone marrow is either invaded with blasts or hypoplastic. Neutropenia allows continuous bacterial invasion of the bowel, perpetuating the lesion, with possible necrosis and perforation $[3,15]$.

\section{Conclusion}

In our study, the rate of typhlitis among leukemic children was $4.5 \%$; however, the mortality rate was $20 \%$. Thus, rapid identification and timely, aggressive medical intervention are necessary to reduce the morbidity and mortality from typhlitis.

\section{References}

-1 Tiseo M, Gelsomino F, Bartolotti M, Barili MP, Ardizzoni A: Typhlitis during secondline chemotherapy with pemetrexed in nonsmall cell lung cancer (NSCLC): a case report. Lung Cancer 2009;65:251-253.

-2 Moran H, Yaniv I, Ashkenazi S, Schwartz M, Fisher S, Levy I: Risk factors for typhlitis in pediatric patients with cancer. J Pediatr Hematol Oncol 2009;31:630-634.

-3 Dietrich CF, Hermann S, Klein S, Braden B: Sonographic signs of neutropenic enterocolitis. World J Gastroenterol 2006;12:13971402.

-4 McCarville MB, Adelman CS, Li C, Xiong X, Furman WL, Razzouk BI, Pui CH, Sandlund JT: Typhlitis in childhood cancer. Cancer 2005; 104:380-387.

5 Ullery BW, Pieracci FM, Rodney JR, Barie PS: Neutropenic enterocolitis. Surg Infect (Larchmt) 2009;10:307-314.

6 Mullassery D, Bader A, Battersby AJ, Mohammad Z, Jones EL, Parmar C, Scott R, Pizer BL, Baillie CT: Diagnosis, incidence, and outcomes of suspected typhlitis in oncology patients: experience in a tertiary pediatric surgical center in the United Kingdom. J Pediatr Surg 2009;44:381-385.
7 Gorschlüter M, Mey U, Strehl J, Ziske C, Schepke M, Schmidt-Wolf IG, Sauerbruch T, Glasmacher A: Neutropenic enterocolitis in adults: systematic analysis of evidence quality. Eur J Haematol 2005;75:1-13.

8 Williams N, Scott AD: Neutropenic colitis: a continuing surgical challenge. Br J Surg 1997;84:1200-1205.

-9 Furonaka M, Miyazaki M, Nakajima M, Hirata S, Fujitaka K, Kondo K, Yokojama A, Maeda H, Kohno N: Neutropenic enterocolitis in lung cancer: a report of two cases and a review of the literature. Intern Med 2005; 44:467-470.

10 Sigirci A, Akinci A, Ozgen O, Ozen O: Neutropenic enterocolitis (typhlitis) associated with infectious mononucleosis. Pediatr Radiol 2006;36:155-157.
Cartoni C, Dragoni F, Micozzi A, Pescarmona E, Mecarocci S, Chirletti P, Petti MC, Meloni G, Mandelli F: Neutropenic enterocolitis in patients with acute leukemia: prognostic significance of bowel wall thickening detected by ultrasonography. J Clin Oncol 2001;19: 756-761.

12 Lee JH, Lim GY, Im SA, Chung NG, Hahn ST: Gastrointestinal complications following hematopoietic stem cell transplantation in children. Korean J Radiol 2008;9:449-457.

13 Takaoka E, Kawai K, Ando S, Shimazui T, Akaza H: Neutropenic colitis during standard dose combination chemotherapy with nedaplatin and irinotecan for testicular cancer. Jpn J Clin Oncol 2006;36:60-63.

14 Siegel MJ, Friedland JA, Hildebolt CF: Bowel wall thickening in children: differentiation with US. Radiology 1997;203:631-635.

-15 Katz JA, Wagner ML, Gresik MV, Mahoney DH Jr, Fernbach DJ: Typhlitis: an 18-year experience and postmortem review. Cancer 1990;65:1041-1047.

16 Özgen Ü, Üzüm İ, Mizrak B, Saraç K: ‘Typhlitis' in rectum. Pediatr Int 2010;52:e32-e33. 